\title{
Modeling Chaotic Current Oscillations in Semi-Insulating GaAs with Rate-Equations of Impact Ionization and Field-Enhanced Trapping
}

\author{
H. A. Albuquerque ${ }^{1}$, R. L. da Silva ${ }^{1}$, R. M. Rubinger ${ }^{2}$, A. G. de Oliveira ${ }^{1}$, G. M. Ribeiro ${ }^{1}$, and W. N. Rodrigues ${ }^{1}$ \\ ${ }^{1}$ Departamento de Física, Instituto de Ciências Exatas, Universidade Federal de Minas Gerais, \\ Caixa Postal 702, 30123-970, Belo Horizonte, MG, Brazil \\ ${ }^{2}$ Departamento de Física e Química, Instituto de Ciências, \\ Universidade Federal de Itajubá, Caixa Postal 50, 37500-903, Itajubá, MG, Brazil
}

\author{
Received on 4 April, 2005
}

\begin{abstract}
We investigated the effect of adding the field-dependent recombination process, namely field-enhanced trapping, to the generation-recombination processes of charge carriers that model current oscillations in semiconductors. The main new features arising from this modification are identified in bifurcation diagrams with the electric field as the control parameter. The characteristic of the bifurcation diagrams is a function of impurity energy. Thus, we generated a set of bifurcation diagrams for a range of the impurity energy and applied bias. The energy dependence of the bifurcation diagrams is discussed considering the context of the competition between the generation-recombination mechanisms impact ionization and field-enhanced trapping.
\end{abstract}

Keywords: generation-recombination processes; current oscillations in semiconductors; bifurcation diagrams

\section{INTRODUCTION}

Low-frequency current oscillations (LFO) in semiinsulating (SI) GaAs were observed in many works since the 60's [1-4]. Theoretical models have been proposed to explain the LFOs and the chaotic behavior in GaAs, $(\mathrm{Si})$ and in other semiconductors [5-7].

In a recent work [4], we carried out analyses on experimental LFO, measured in semi-insulating GaAs samples. Those analyses were carried out considering some methods of nonlinear dynamics analysis applied to time series [8,9]. Such procedures work as if the system under investigation were "black boxes", i.e. systems in which there is no direct access to their dynamical equations. This means that it cannot provide all the needed information contained in its dynamical equations. Besides, for experimental systems contaminated by noise, as in our case, time series measures are inconclusive. In order to understand experimental systems like ours $[3,4]$, that present nonlinear characteristics and even deterministic chaos, we are now trying to develop the theory underlying the physical processes responsible for these chaotic characteristics. This is carried out by defining and building the system's dynamical equations and numerically integrating them to study its dynamics.

Current oscillations in semiconductors are in most cases originated from Negative Differential Conductivity (NDC), which occurs due to various physical mechanisms. The recombination instability is one of them, and is closely related with field-enhanced trapping effect. It was suggested that this effect could lead the system to NDC, and this type of NDC was observed in GaAs Si [10-12]. This negative type of nonlinearity leads to the nucleation and propagation of field domains. Current oscillations occur as a result of cyclic propagation of domain nucleating at the cathode and vanishing at the anode. A review about field domains in GaAs Si can be found in Ref. [10].

The present work concerns the investigation of the effect of including in the rate-equations firstly proposed by Schöll for the generation-recombination (g-r) processes of charge carriers [5], the field-dependent recombination process, namely field-enhanced trapping $[11,12]$. These equations were applied to explain the self-generated chaos in semiconductors. In this model, Schöll used the field-dependent impact ionization process in a two-impurity levels model plus the conduction band. In our model, we included the field-enhanced trapping process. The main new features arising from this modification are identified in bifurcation diagrams for the electric field as a function of the control parameter, i.e. the applied bias. The characteristics of the bifurcation diagrams of the modified model are impurity energy dependent. We generated a set of bifurcation diagrams for a range of the impurity energy and applied bias. The energy dependence of the bifurcation diagrams is discussed considering the competition between the g-r mechanisms impact ionization and field enhanced trapping.

\section{MODELING THE PHYSICAL SYSTEM}

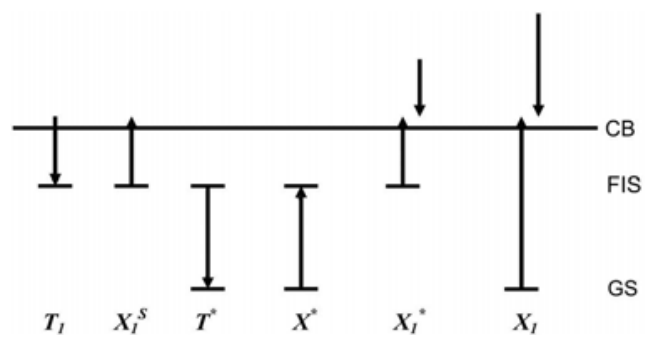

FIG. 1: Generation-recombination model involving the conduction band (CB) and the defects levels - ground state (GS) and the first ionized state (FIS) [5].

The model studied in this work was initially proposed by Schöll [5]. The model consists of three rate equations for the g-r processes of charge carrier in a simplified extrinsic n-type 
semiconductor. The dynamic equations represent two defect energy levels, i.e., its ground and first ionized states, and the conduction band. The valence band is omitted because the recombination rates involving it are negligible when compared with the processes involving the impurity states and the conduction band. The scheme of this model is represented in Fig. 1 , where the g-r coefficients are shown. The up arrows represent the generation and the down arrows, the recombination processes of the electrons. Some g-r coefficients depend, on the electric field [11-13]. In the present model the coefficients of generation processes $\left(X_{1}^{*}, X_{1}\right)$, due impact ionization, and of recombination process $\left(T_{1}\right)$, due field-enhanced trapping, depend on the electric field. All other coefficients were considered field independent. The impact ionization phenomenon is assumed to follow the Schockley's model [13], which is given by $X_{1}^{*}, X_{1} \propto \exp \left[-\mathrm{E}_{0} /(e \lambda E)\right]$, where $\mathrm{E}_{0}$ is the threshold ionization energy, $e$ is the electron charge, $\lambda$ is the mean free path, and $E$ is the electric field. The field-enhanced trapping phenomenon is assumed to follow the same model except by a signal change and the use of proper carriers on the differential equation since it is a recombination process. Thus $T_{1}$ is given by $T_{1} \propto \exp \left[-\mathrm{E}_{1} /(e \lambda E)\right]$, where $\mathrm{E}_{1}$ is the threshold capture energy $[11,12]$. The rate equations can then be built from Fig. 1 , as well the constitutive equations [5-7].

Following Schöll [5], the set of differential rate equations for the normalized variables $\left(n, n_{1}, \varepsilon\right)$ is:

$$
\begin{gathered}
\dot{n}=X_{1}^{S} n_{2}-T_{1}^{n} p_{t}+X_{1} n n_{1}+X_{1}^{*} n n_{2}, \\
\dot{n}_{1}=T^{*} n_{2}-X^{*} n_{1}-X_{1} n n_{1}, \\
\dot{\varepsilon}=J-n V(\varepsilon) .
\end{gathered}
$$

Where $n$ is the carrier density in the conduction band, $n_{1}$ is the trapped carrier density in the donor ground state and $\varepsilon$ is the electrical field. The electron density of the excited state, $n_{2}$, was eliminated due to the charge neutrality condition given by $n_{2}=N_{D}^{*}-n_{1}-n$. The unoccupied defect density, $p_{t}$, is given by $p_{t}=N_{A}+n$. The effective donor density and the acceptor density are represented by $N_{D}^{*}$ and $N_{A}$, respectively. The coefficient $X_{1}^{S}$ stands for thermal ionization coefficient, $T^{*}$ and $X^{*}$ are the coefficients for transitions from the ground state to the excited state and vice versa, respectively (Fig. 1). The coefficients $X_{1}$ and $X_{1}^{*}$ are the generation coefficients via impact ionization for the ground and excited state, respectively, which are electric field dependent following the Schockley's model. $T_{1}$ is the recombination coefficient via field-enhanced trapping, which is electric field dependent. The coefficients $X_{1}^{S}, T^{*}$ and $X^{*}$ are considered independent of the electric field. The dimensionless parameter $V(\varepsilon)$ is the field-dependent drift velocity and will be defined in the next section. The control parameter, $\varepsilon_{0}$, is given by the steady-state current-density-field characteristic when Eqs. (1) is equal to zero, i.e., $J=n V\left(\varepsilon_{0}\right)$.
In this work we have studied normalized versions of Eqs. (1), i.e. electric field $\varepsilon$, carrier density $n$ in the conduction band and trapped carrier density $n_{1}$ in the donor ground state were normalized according to Refs. [5-7]. We have performed a study of bifurcation and chaos of electric field oscillations obtained by numerical integration of the set of equations, Eqs. (1). The control parameter considered for the bifurcation diagrams is the applied bias, i.e., $\varepsilon_{0}$.

\section{NUMERICAL RESULTS}

By integrating Eqs. (1), tuning the control parameter $\varepsilon_{0}$ and the capture energy $E_{1}$, a period doubling bifurcation route to chaos and other types of bifurcation were found.

The values of the dimensionless g-r coefficients used for integrating the set of equations, Eqs. (1), are shown in Table 1. The impact ionization coefficients $X_{1}$ and $X_{1}^{*}$ have a nonlinear dependence with the normalized electric field $\varepsilon$ and are given by: $X_{1}=X_{1}^{0} \exp \left(-6.0 E_{t} / \varepsilon\right)$ and $X_{1}^{*}=X_{1}^{* 0} \exp \left(-1.5 E_{t} / \varepsilon\right)$, respectively. The energy $E_{t}$ is a normalized impurity groundstate value and is unitary. The field-enhanced trapping coefficient $T_{1}$ has a nonlinear dependence with the normalized electric field $\varepsilon$ and is given by: $T_{1}=T_{1}^{0} \exp \left(-E_{1} / \varepsilon\right)$. The $N_{A} / N_{D}^{*}$ ratio is a compensation factor and is equal 0.3. The parameter $r_{2}$ is a saturation value that is related to the dimensionless empirical drift velocity [14] $V(\varepsilon)$ as: $V(\varepsilon)=\arctan \left(r_{2} \varepsilon\right) / r_{2}$. Its value is equal 0.3 . These parameters and coefficients depend on the semiconductor material and sample geometry. Schöll [6] worked with a set of parameters that he proposed for highpurity n-type direct gap materials with hydrogen-like shallow donors presenting " $\mathrm{S}$ " shaped negative differential conductivity (SNDC) at low temperatures. For comparison of the similarities we keep the same values of the parameters of Schöll's work. The only changes considered regard the threshold energies.

TABLE I: Dimensionless g-r coefficients for the Eqs. (1). After Schöll [5-7].

\begin{tabular}{|l|l|l|l|l|l|}
\hline$T_{1}^{0}$ & $T^{*}$ & $X_{1}^{S}$ & $X^{*}$ & $X_{1}^{0}$ & $X_{1}^{* 0}$ \\
\hline $10^{-2}$ & $10^{-5}$ & $10^{-7}$ & $10^{-7}$ & $5 \times 10^{-4}$ & $10^{-2}$ \\
\hline
\end{tabular}

For values presented in Table 1 and for $E_{1}$ equal to $1.7 E_{t}$ we obtained the bifurcation diagram shown in Fig. 2. The horizontal axis is the control parameter $\varepsilon_{0}$ and the vertical axis is the sequence of maxima for the electric field time series. In the range of $\varepsilon_{0}$ showed in Fig. 2, we found only electric field oscillations with fundamental period (period-1). For values of $\varepsilon_{0}$ below 38 and above 43 , the system, represented by Eqs. 1, converge to fixed points.

Figure 3 shows bifurcation diagram for $E_{1}=1.55 E_{t}$. Here the system, Eqs. (1), bifurcates from period-1 to period- 2 with two fundamental frequency and return to period- 1 , for $\varepsilon_{0}$ in the range 50.0 to 55.2. Fig.4 (a) shows bifurcation diagram for $E_{1}=1.5 E_{t}$. Here the system presents a period-doubling bifurcation cascade followed by period-halving cascade. In 


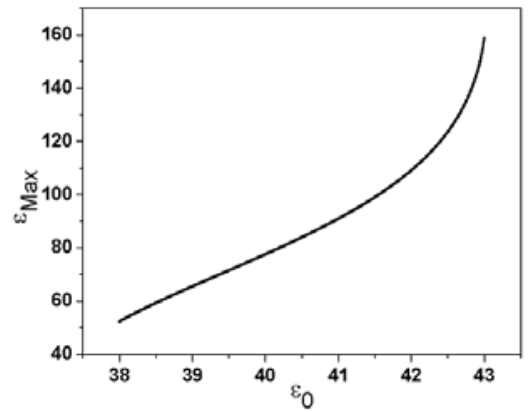

FIG. 2: Bifurcation diagram for $E_{1}=1.7 E_{t}$.

Fig. 4 (b) we present an enlargement of the Fig. 4 (a) in the range 56.15 to 57.20 for $\varepsilon_{0}$. Here we have period-doubling bifurcation and interior crisis. This type of bifurcation is characterized by abrupt changes from chaotic cycle to a period-1 cycle. Fig. 5 shows bifurcation diagram for $E_{1}=1.0 E_{t}$. Here the system, Eqs. (1), bifurcates from period-1 to period-2 followed by period- 4 and returns to period- 2 and bifurcates again following a period-doubling bifurcation. This bifurcation sequence, i.e., period- 1 to period- 2 to period- 4 to period-2, is observed in our experimental measurements of current oscillations as we can observe in Fig. 6.

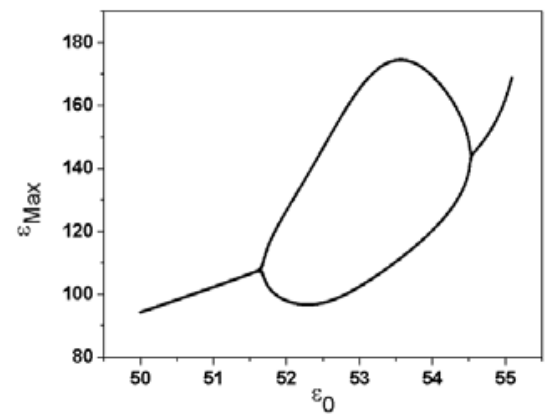

FIG. 3: Bifurcation diagram for $E_{1}=1.55 E_{t}$.

Figure 7 shows bifurcation diagram for $E_{1}=0.1 E_{t}$. Here, the sequence period- 1 to period- 2 to period- 4 to period- 2 disappeared in the second branch, compare with Fig. 5, and a same sequence emerged in the third branch followed by a normal period-doubling bifurcation.

In our model, we generated electric field oscillations instead of current oscillations as in experimental measurements [1-4]. However, the relation between the variables can be obtained by the equation $J=n(\varepsilon) V(\varepsilon)$, where $n(\varepsilon)$ is the dynam-
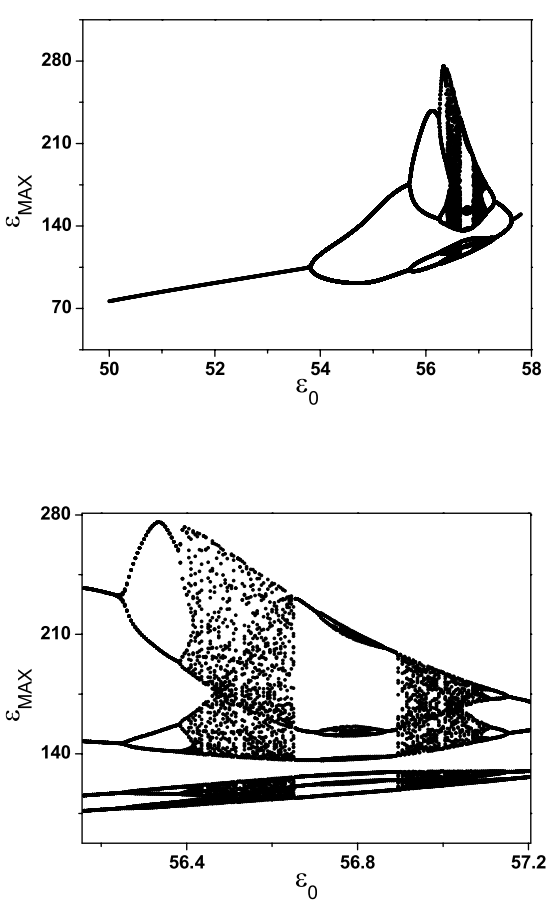

FIG. 4: Bifurcation diagram for $E_{1}=1.5 E_{t}$.

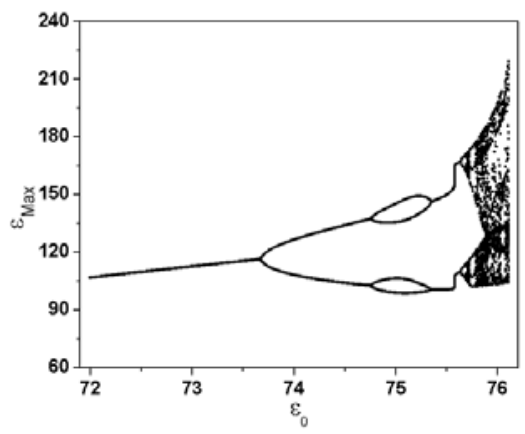

FIG. 5: Bifurcation diagram for $E_{1}=1.0 E_{t}$.

ical variable presented in Eqs. (1) and $V(\varepsilon)$ is the dynamical drift velocity as defined above.

All variables and parameters in Eqs. (1) and Table 1 are normalized. The carrier densities are normalized by effective donor density $N_{D}^{*}=N_{D}-N_{A}$, all lengths by the effective Debye length $L_{D}=\left(D_{0} \tau_{M}\right)^{1 / 2}$, the time by the effective dielectric relaxation time $\tau_{M}=\varepsilon_{S} /\left(4 \pi e \mu_{0} N_{D}^{*}\right)$, and the electric field by $k T / e L_{D}$, where $N_{D}, N_{A}$ are the donor and compensating acceptor concentrations, respectively, $\mu_{0}$ and $D_{0}$ are the low-field mobility and the diffusion constant, and $\varepsilon_{S}$ is the static dielectric constant. For the sample of Fig. 6 at $300 \mathrm{~K}$ with $\mu_{0}=670$ $\mathrm{cm}^{2} / \mathrm{Vs}, \varepsilon_{S}=12.9, N_{D}^{*}=10^{19} \mathrm{~cm}^{-3}$ and trap level at $0.7 \mathrm{eV}$, $\tau_{M} \sim 10 \mu \mathrm{s}$ and $k T E_{t} / e L_{D} \sim 1 \mathrm{~V} / \mathrm{cm}$. 


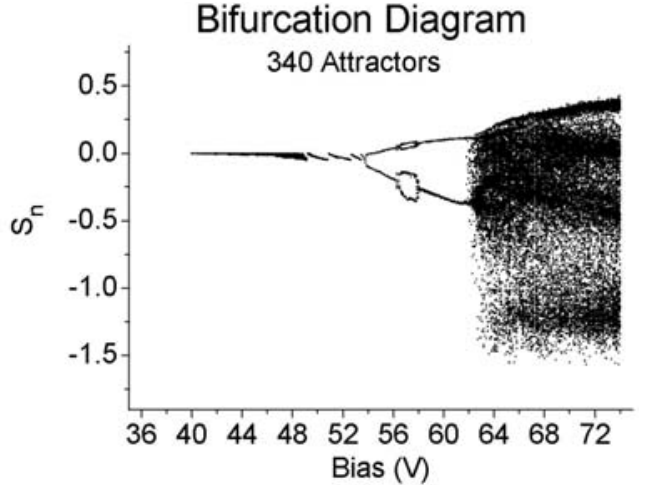

FIG. 6: Bifurcation diagram for the minima of the current time series as a function of the applied bias in the sample [3].

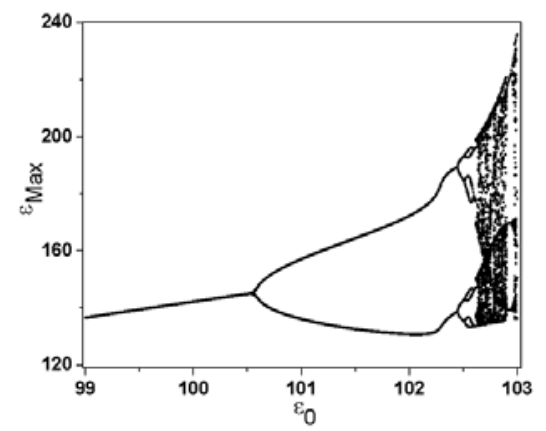

FIG. 7: Bifurcation diagram for $E_{1}=0.1 E_{t}$.

It was found that the recombination instability, fieldenhanced trapping, with generation instability, impact ionization, brought the system, Eqs. (1), to exhibit not only current oscillations with fundamental period, but also those with var- ious kind of period including chaos, which were associated with motion of domains [10]. The complexity of the bifurcation diagrams, presented here, is associated with the trap energy level responsible for the field-enhanced trapping effect. For high value of $E_{1}$, Fig. 2, the system showed a periodic behavior for all values of electric field. In this case, the field-enhanced trapping effect is not a predominant effect and can be negligible. For values between $1.55 E_{t} \leq E_{1} \leq 0.3 E_{t}$, Fig. 3-5, we found a variety of bifurcation including perioddoubling, crisis and bifurcation with the sequence: period-1 period- 2 - period- 4 - period-2. In this range of $E_{1}$ the fieldenhanced trapping effect dominates or there is a competition with impact ionization effect. Below $0.3 E_{t}$, Fig. 7 , the system returns to period-doubling bifurcation where the impact ionization effect dominates.

\section{SUMMARY}

We included, in a model of rate-equations for the g-r processes of charge carriers, the field-enhanced trapping effect. We obtained a set of bifurcation diagrams with electric field and trap energy level as control parameters. Depending on the trap energy level, the system can bifurcate by perioddoubling, crisis, period- 1 to period- 4 and return to period- 1 or no bifurcations at all. The field-enhanced trapping effect plays an important role in the process of bifurcation through the competition with the impact ionization effect. Further studies will be carried out in this model to better elucidate its relation with experimental data.

\section{Acknowledgement}

The authors would like to acknowledge the Brazilian agencies $\mathrm{CNPq}$ and FAPEMIG for financial support.
[1] D. C. Northrop, P. R. Thornton, and K. E. Trezise, Solid State Electron. 7, 17 (1964).

[2] G. N. Maracas, W. Porod, D. A. Johnson, and K. Ferry, Physica B134, 276 (1985).

[3] R. L. da Silva, H. A. Albuquerque, R. M. Rubinger, A. G. de Oliveira, and G. M. Ribeiro, Physica D 194, 166 (2004).

[4] R. M. Rubinger, R. L. da Silva, A. G. de Oliveira, G. M. Ribeiro, and H. A. Albuquerque, Chaos 13, 457 (2003).

[5] E. Schöll, Phys. Rev. B 34, 1395 (1986).

[6] E. Schöll, Nonequilibrium Phase Transitions in Semiconductors, Springer-Verlag, Berlin, 1987.

[7] E. Schöll, Nonlinear Spatio-Temporal Dynamics and Chaos in Semiconductors, Cambridge Nonlinear Science Series 10, Cambridge University Press, Cambridge, 2001.
[8] H. D. I. Abarbanel, R. Brown, J. J. Sidorowich, and L. Sh. Tsimring, Rev. Mod. Phys. 65, 1331 (1993).

[9] R. Hegger, H. Kantz, and T. Schreiber, Chaos 9, 413 (1999).

[10] A. Neumann, J. Appl. Phys. 90, 1 (2001).

[11] R. M. Rubinger, A. G. de Oliveira, G. M. Ribeiro, J. C. Bezerra, M. V. Moreira, and H. Chacham, J. Appl. Phys. 88, 6541 (2000).

[12] H. A. Albuquerque, A. G. de Oliveira, G. M. Ribeiro, R. L. da Silva, W. N. Rodrigues, M. V.B. Moreira, and R. M. Rubinger, J. Appl. Phys. 93, 1647 (2003).

[13] W. Schockley, Solid-State Electron. 2, 35 (1961).

[14] R. M. Westervelt, and S. W. Teitsworth, J. Appl. Phys. 57, 5457 (1985). 